\title{
A Case of Paediatric Sudden Visual Loss
}

\author{
Verrotti di Pianella V, Damiano B, Basilone A, Sciacovelli M, Dipasquale L, Cinalski J, Patisso I, \\ Pedico A, Di Florio L, Merola T, Marseglia A, Rutigliano I*, Pellegrino M, Pettoello Mantovani M \\ and Sacco $M$
}

Pediatric Unit, IRCCS Casa Sollievo della Sofferenza, San Giovanni Rotondo - Residency Program in Pediatrics, University of Foggia Medical School, Italy

*Corresponding author: Rutigliano Irene, Pediatric Unit, IRCCS Casa Sollievo della Sofferenza, San Giovanni Rotondo Residency Program in Pediatrics, University of Foggia Medical School, Italy

\section{ARTICLE INFO}

Received: 幽 September 18, 2020

Published: 彗 September 29, 2020

Citation: Verrotti di Pianella V, Damiano B, Basilone A, Sciacovelli M, Dipasquale L, Cinalski J, et al. A Case of Paediatric Sudden Visual Loss. Biomed J Sci \& Tech Res 30(5)-2020. BJSTR. MS.ID.005010.

Abbreviations: MS:Multiple Sclerosis; NMOSD: Neuromyelitis Optic Spectrum Disorder; MRI: Magnetic Resonance Imaging; CNS: Central Nervous System; TPE: Therapeutic Plasma Exchange; ON: Optic Neuritis; TM: Transverse Myelitis

\section{ABSTRACT}

\begin{abstract}
A Sudden Visual Loss
We admitted to our department M., a 8 years old girl, for sudden bilateral vision loss. The onset of visual symptoms had been preceded by an important and self-healing headache. A bilateral papilledema was detected, with the evidence of a severe impairment of visual acuity. Laboratory blood tests were normal, except for ANA positivity. Optic nerve transorbital sonography and Brain Magnetic Resonance (MRI) revealed bilateral thickening of the optic nerves in the retrobulbar tract. The Cerebrospinal Fluid (CSF) examination was normal and the oligoclonal bands were negative. Serology tests revealed Anti-MOG antibodies negativity, while anti-AQP4 antibodies were positive. Based on clinical, laboratory and MRI findings, diagnosis of Optic Neuromyelitis (NMO) was made. NMO is an inflammatory demyelinating disease of the Central Nervous System (CNS), based on an autoimmune pathogenesis. It is traditionally characterized by optic neuritis and transverse myelitis. First line therapy is based on high dose pulse steroids; in case of treatment failure, second- line therapy with Therapeutic Plasma Exchange (TPE) can be proposed.
\end{abstract}

Keywords: Optic neuritis; Inflammatory; Spinal Cord; Vomiting; Vision Loss; Papillae

\section{Introduction}

Optic neuromyelitis (NMO) is a demienilizing disease traditionally characterized by optic neuritis and transverse myelitis [1], also known as Devic's disease, formerly considered as a variant of Multiple Sclerosis (MS) [2]. Nowadays, NMO is included in a wider set of pathological findings referred to "Neuromyelitis Optic Spectrum Disorders" (NMOSDs), a group of inflammatory and demyelinating disorders of the Central Nervous System (CNS), differentiated from multiple sclerosis [3].NMO affects adults mainly in the fourth and fifth decade of life; in children the typical age of onset is around 10-12 years. According to the sites affected by the disease (optic nerve, chiasm, hypothalamus, medulla oblongata, subcortical white matter and spinal cord) a wide range of symptoms can be observed: motor, visual, but also, in case of involvement of the postrema area, hiccups and vomiting $[3,4]$.The most common clinical onset is related to optic neuritis, which manifests itself with acute or subacute (frequently bilateral) loss of visual acuity, ocular pain, worsening central scotoma and reduced perception of colors [1].We report the description of the clinical case of a patient, who came to our department for sudden vision loss, subsequently revealed, at the end of the appropriate investigations, as a NMO.

\section{Case Report}

M. is a 8 years old female patient, admitted to our department for sudden bilateral vision loss.A suspected erythromelalgia is detected from her past history, with ANA positivity (1:160), but without symptoms for a long time. The day before the onset of the visual symptoms, the little girl had a closed-head injury, followed by a rapidly self-healing headache. Therefore M. was examined 
by an ophthalmologist who detected bilateral papilledema so that the patient was referred to the Ophthalmology division for further evaluation. Routine blood tests were done, all of which were normal. The visual acuity test was severely impaired (VOS 0/10 e VOD 1/10); fundus examination detected blurred margins papillae; visual evoked potential highlighted reduced amplitude and increased latency. Moreover, optic nerve transorbital sonography and brain Magnetic Resonance (MRI),revealed bilateral thickening of the optic nerves in the retrobulbar tract while spinal MRI showeda normal picture. Then M. was referred to our department for further evaluation. Neurological physical examination was normal; blood tests, with complete blood count, ESR, PCR, coagulation function, immunoglobulins, virological screening, thyroid function and peripheral blood smear test, were all normal. ANA positivity was confirmed with negativity of the remaining autoimmunity. The Cerebrospinal Fluid (CSF) examination was normal and the oligoclonal bands, were negative. Anti-MOG antibodies examination was negative, while Anti-AQP4 antibodies was positive. Based on the above-mentioned clinical, laboratory and MRI findings, the diagnostic criteria for NMOSD were met and so the diagnosis of NMO was made. Therefore, M. started intravenous boluses of Methylprednisolone (1 gr/day for 3 days)with only a little vision improvement.For this reason the girl was referred to the regional reference hospital for startingthe second- line therapy with Therapeutic Plasma Exchange (TPE).

\section{Discussion}

NMO is an inflammatory demyelinating disease of CNS, based on an autoimmune pathogenesis. The classic clinical presentation is given by Optic Neuritis (ON) and Transverse Myelitis (TM); however the clinical onset can be also characterized by other clinical manifestations, such as area postrema syndrome and acute brain stem syndromes, justifying the term NMOSD [3]. AntiAQP4 antibodies are a disease-specific biomarkers of NMOSD [5] and the seropositivity for this antibody represents a diagnostic criteria for NMOSDs [6] and facilitate the differential diagnosis with other inflammatory CNS demyelinating disease. The presence of at least one "main clinical feature" (optic neuritis, acute myelitis, area postrema syndrome,brain stem syndromes, symptomatic narcolepsy or acute diencephalic syndrome) associated with brain MRI lesions and the seropositivity for AQP-4 antibodies allows the diagnosis of NMOSD [6,7]. In the absence of anti-AQP4 antibodies, diagnosis of NMOSD is possible, although relatively more complex because at least two main clinical criteria are needed, always supported by a compatible neuroimaging findings. M.'s clinical history began with a sudden and important decrease in vision: the finding of bilateral edema of the papilla at the FOO and abnormalities both in the ETG of the optic nerves and in the examination of the VEPs guided our diagnostic hypothesis of NMO.
Final diagnosis was made on the basis of brain MRI which showed an evident inflammatory lesion of both optic nerves and the AQP-4 seropositivity. On the basis of the investigations carried out, we exclude a neoplastic-compressive etiology as a cause of papillary edema: the brain MRI, in fact, was negative for masses and / or hemorrhagic spreads. Intracranial MRI angiography allowed to exclude relevant vascular anomalies. We paid attention to possible differential diagnoses (infectious diseases, vasculitis, demyelinating diseases of the CNS, neurological complications of lupus, neurosarcoidosis) by testing liquor, systemic inflammation indices, serology for the main viral and bacterial agents, autoimmunity, dosage of the ACE enzyme, anti-MOG and anti AQP4 antibodies. The absence ofboth oligoclonal bands in CSF and CNS lesions on MRI, allowed us to exclude MS according to the 2017 McDonald criteria [7], which could manifest itself at the onset just like Optic Neuritis (ON). Also ADEM includes ON among its clinical presentation, however we discarded this hypothesis due to the absence of encephalitic-like clinical features and for the absence of multifocal brain lesions on MRI.

The finding of anti-Aquaporin-4 antibodies was decisive: in association with the clinical and radiological features of $\mathrm{ON}$, despite the absence of spinal cord lesions, allowed us to formulate the diagnosis of NMOSD. Therefore M. immediately started the first line therapy with boluses of Methyilprednisolone (1 gr/day for 3 days), followed by oral Prednisolone with progressive décalage in 4 weeks. Despite the early and generous steroid therapy, M. showed only a very slight improvement in visual function, so we referred her to the regional reference center to start the second-line therapy with cycles of plasmapheresis. After two months, M came back to our department and we evaluated an important improvement in visual acuity only inthe right eye with a persistent visual disability in the left one.

\section{References}

1. Melinda Y Chang, Stacy L Pineles (2017) Pediatric Optic Neuritis. Seminars in Pediatric Neurology. 24(2): 122-128.

2. Kliegman Stanton, St Geme, Schor Behrman (2012) Pediatria di Nelson. Ed Elsevier 2.

3. Grace Y Gombolay, Tanuja Chitnis (2018) Pediatric Neuromyelitis Optic Spectrum Disorders. Multiple Sclerosis and Related Disorders 85(2): 177-189.

4. Zuccotti G V (2016) Manuale di Pediatria. Clinical practice.

5. Gombolay GY, Chitnis T (2018) Pediatric Neuromyelitis Optica Spectrum Disorders. Curr Treat Options Neurol. 20(6): 19.

6. Mark Borchert, Grant T Liu, Stacy Pineles, Amy T Waldman (2017) Pediatric Optic Neuritis: What Is New. Journal of Neuro-Ophthalmology 37(Suppl): S14-S22.

7. Thompson AJ, Banwell BL, Barkhof F, William M Carroll, Timothy Coetzee, et al. (2018) Diagnosis of multiple sclerosis: 2017 revisions of the McDonald criteria. Lancet Neurol 17(2):162-173. 
ISSN: 2574-1241

DOI: $10.26717 /$ BJSTR.2020.30.005010

Rutigliano I. Biomed J Sci \& Tech Res

(c) (i) This work is licensed under Creative Submission Link: https://biomedres.us/submit-manuscript.php

\begin{tabular}{ll} 
BIOMEDICAL & Assets of Publishing with us \\
RESEARCHES & - Global archiving of articles \\
\hline Immediate, unrestricted online access \\
\hline - Rigorous Peer Review Process
\end{tabular}

